\title{
ERRATUM
}

B. Egger · P. Ladurner $\cdot$ K. Nimeth $\cdot$ R. Gschwentner

R. Rieger

\section{The regeneration capacity of the flatworm Macrostomum lignano-on repeated regeneration, rejuvenation and the minimal size needed for regeneration}

Published online: 29 April 2006

C) Springer-Verlag 2006

\section{Dev Genes Evol (2006)}

Table 1 contained errors in the original publication.

The online version of the original article can be found at: http://dx. doi.org/10.1007/s00427-006-0069-4

B. Egger $(\bowtie) \cdot$ P. Ladurner · K. Nimeth · R. Gschwentner · R. Rieger

Ultrastructural Research and Evolutionary Biology, Institute of

Zoology, University of Innsbruck,

Innsbruck, Austria

e-mail: bernhard.egger@uibk.ac.at

Tel.: +49-512-5076192

Fax: +49-512-5072930 
Table 1 The correct version of Table 1 is shown below

\begin{tabular}{|c|c|c|c|c|c|c|}
\hline & & cutting level & $\begin{array}{l}\text { number of } \\
\text { specimens }\end{array}$ & $\begin{array}{l}\% \text { fully } \\
\text { regenerated }\end{array}$ & $\begin{array}{l}\text { days until full } \\
\text { regeneration }\end{array}$ & $\begin{array}{l}\text { days until death, if no } \\
\text { full regeneration }\end{array}$ \\
\hline \multirow{17}{*}{$\begin{array}{l}\text { transversal } \\
\text { amputation }\end{array}$} & anterior & just anterior of brain & 11 & 81.82 & about 2 weeks & \\
\hline & regeneration & just anterior of eyes & 5 & 0 & & \\
\hline & & $\begin{array}{l}\text { anterior half of } \\
\text { pharynx }\end{array}$ & 7 & 0 & & more than 9 weeks \\
\hline & & middle of pharynx to gonads & 114 & 0 & & more than 9 weeks \\
\hline & & tail plate & 13 & 0 & & up to 8 days \\
\hline & $\begin{array}{l}\text { posterior } \\
\text { regeneration }\end{array}$ & $\begin{array}{l}\text { posterior end of } \\
\text { rostrum }\end{array}$ & 12 & 0 & & up to 3 days \\
\hline & & posterior of eyes & 13 & 0 & & up to 3 days \\
\hline & & mid of pharynx & 15 & 0 & & up to 6 days \\
\hline & & $\begin{array}{l}\text { middle to end of } \\
\text { pharynx }\end{array}$ & 35 & 14.29 & about 25 days & up to 16 days \\
\hline & & $\begin{array}{l}\text { end of pharynx, } \\
\text { a little bit of gut }\end{array}$ & 50 & 58.00 & about 3 weeks & $\begin{array}{l}\text { up to } 14 \text { days most within } \\
7 \text { days }\end{array}$ \\
\hline & & possibly anterior tip of testes & 11 & 90.91 & about 17 days & 2 days \\
\hline & & mid of gonads & 5 & 100.00 & about 2 weeks & \\
\hline & & tail plate & 32 & 100.00 & $6-10$ days & \\
\hline & $\begin{array}{l}\text { two-sided } \\
\text { regeneration }\end{array}$ & $\begin{array}{l}\text { posterior of eyes and } \\
\text { at tail plate }\end{array}$ & 6 & 0 & & more than 3 weeks \\
\hline & & $\begin{array}{l}\text { in rostrum and at } \\
\text { gonad level }\end{array}$ & 1 & 100.00 & about 4 weeks & \\
\hline & $\begin{array}{l}\text { repeated } \\
\text { regeneration }\end{array}$ & $\begin{array}{l}\text { between testes and tail } \\
\text { plate }\end{array}$ & 20 & 100.00 & cut 29 times du & Iring 12 months \\
\hline & juveniles & half of body to tail plate & 24 & 62.50 & 2-4 weeks & \\
\hline \multirow[t]{2}{*}{$\begin{array}{l}\text { oblique } \\
\text { amputation }\end{array}$} & $\begin{array}{l}\text { anterior } \\
\text { regeneration }\end{array}$ & between eyes & 9 & 0 & & \\
\hline & $\begin{array}{l}\text { posterior } \\
\text { regeneration }\end{array}$ & between eyes & 9 & 0 & & up to 3 days \\
\hline \multirow{4}{*}{$\begin{array}{l}\text { longitudinal } \\
\text { incision }\end{array}$} & anterior & cut symmetrically & 19 & 36.84 & about 1 week & \\
\hline & regeneration & cut asymmetrically & 28 & 39.29 & about 1 week & \\
\hline & posterior & cut symmetrically & 13 & 53.85 & about 1 week & \\
\hline & regeneration & cut asymmetrically & 15 & 40.00 & about 1 week & \\
\hline
\end{tabular}

